\title{
Homing in on delivery
}

\section{The scientific community now seems convinced that small RNAs will become therapies, if new tools can help these large molecules to make it safely into cells. Monya Baker reports.}

Researchers in the field of small RNAs have been on a wild ride. Twenty years ago, RNA was considered a passive conveyer of information between DNA and protein. Now it is understood that RNA controls and coordinates almost everything that goes on in a cell. From being long unrecognized, then heralded as a convenient tool for screening gene function, small RNAs are now hotly pursued as therapies.

Advocates of using small RNAs to treat disease like to tick off the hurdles that were anticipated but seem to have been cleared: that the computational techniques for predicting appropriate RNA sequences would be overwhelming, that synthetic oligonucleotides would be too expensive to manufacture, that the problems uncovered with earlier RNA drugs would crop up again, and that there would be no way of mitigating 'off-target' (or nonspecific) effects.

The field has moved fast. The complex process by which small RNAs are able to silence genes by targeting complementary messenger RNA molecules for destruction was recognized and named RNA interference (RNAi) in 1998 (ref. 1). In 2004, the first RNAi-based experimental therapies entered clinical trials.

For the past few years, though, researchers

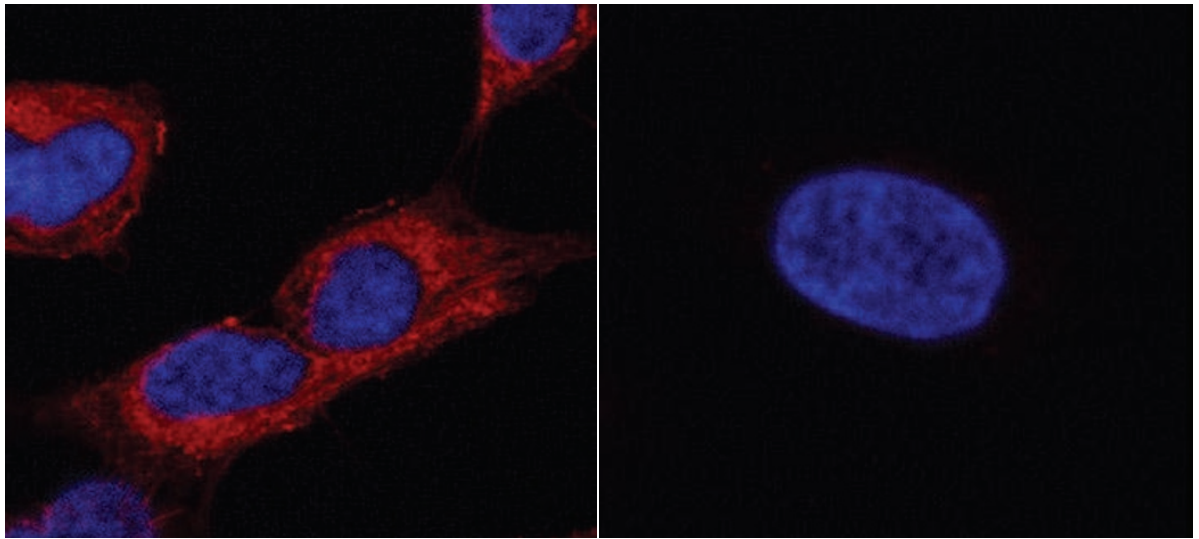

Small RNAs (red) don't usually enter cells by themselves (right) but can when chemically modified (left).

have been stalled by the challenge of delivering small RNA molecules in vivo. "It's still the place where the most important innovations are being made," says Phillip Sharp, a researcher at the Massachusetts Institute of Technology (MIT) in Cambridge and one of the founders of Alnylam Pharmaceuticals in Cambridge, Massachusetts, the first company explicitly founded to harness RNAi. Delivery is also a stumbling block for researchers hoping to use small RNAs to carry out basic research into diseases in living mammals (see 'From tools to therapies').

\section{Getting in}

For many experimental RNA therapies, synthetically produced oligonucleotides are somehow delivered into the desired cells in the body by way of targeting agents, chemical modifications or administration directly to the organ of interest. To test this approach, various clinical trials are in progress, involving many

\section{FROM TOOLS TO THERAPIES}

It's not only clinical researchers who are looking for the best ways to get synthetic oligonucleotides into cells in living mammals. If the delivery problem were solved, says Phillip Sharp, who studies RNA interference (RNAi) at the Massachusetts Institute of Technology (MIT) in Cambridge, the ability to use animals to study physiology and disease would expand dramatically. "You can't make a knockout dog, but you could probe genetic function with RNAi." He is optimistic that in a few years researchers will have worked out ways to exploit RNAi in the immune system and the digestive system, at least. It's an open question whether all tissues will be accessible, he says. "Formulating nanoparticles for certain tissue types is still a pretty sophisticated business."

The challenge of delivering the molecules has not stopped companies from producing in vivo research tools. Those offering products for animal RNAi studies include Ambion, part of Applied Biosystems in Austin, Texas; Exiqon in Vedbaek, Denmark; Integrated DNA Technologies, headquartered in Coralville, lowa; and Thermo Fisher Scientific, headquartered in Waltham, Massachusetts. These products are widely used in mice, but they lack some of the tissue or organ specificity that researchers desire. Companies are listening: in December last year, in what it

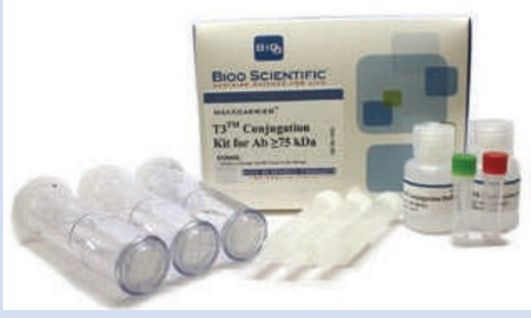

Bioo Scientific's T3 Conjugation Kit can be used to target RNAs to specific cells in the body. announced as the first targeted delivery vehicle to hit the market, Bioo Scientific in Austin launched a kit containing a proprietary linking modality that allows researchers to conjugate RNA molecules of their choice to antibodies, in this way targeting them to specific cells.

This is still a young field, however, and scientists may rightfully fee that the PubMed database is a better source of such technology than product catalogues. In fact, many of the best encapsulation tools are not readily available, given that the tools companies are now seeding therapeutics companies with technologies and expertise, says Mark Behlke, one of the founders of Dicerna Pharmaceuticals in Watertown, Massachusetts. Dicerna is pursuing RNA drugs built on the same platform as some of the research tools offered by Integrated DNA Technologies, of which he is chief scientific officer. Matt Winkler says that he decided to sell Ambion, the research tools company he founded, and launch a therapeutics-focused company after a chance discovery with enormous therapeutic potential. Ambion scientists who were characterizing small RNAs for the company catalogue realized that cancer tissues were surprisingly deficient in certain RNA molecules.

There are other examples. William Marshall co-founded tools company Dharmacon (now owned by Thermo Fisher Scientific) before co-launching miRagen Therapeutics in Boulder, Colorado. Exiqon and Santaris Pharma, headquartered in Hoersholm, Denmark, are commercializing the tool and therapeutic aspects of similar nucleic-acid technologies. And scientists who a few years ago were developing tools are at other companies thinking about the best approaches for clinical trials. M.B. 
companies, including Alnylam, Santaris Pharma, headquartered in Hoersholm, Denmark, and Tekmira Pharmaceuticals in Burnaby, Canada. In another approach, genetic material is delivered not just into cells but into the nucleus to direct the production of small RNA precursors by the cell's gene-transcription machinery. Most techniques involve modified viral vectors, particularly lentiviruses, and clinical trials are under way for treating metastatic melanoma (at Duke University in Durham, North Carolina) and HIV infection (at the City of Hope's Helford Clinical Research Hospital in Duarte, California, with vectors supplied by biotechnology company Benitec of Melbourne, Australia).

The optimal approach depends on both the tissue of interest and the genes targeted, says Mark Kay, who works on both types of delivery mechanism at Stanford University in California. Oligonucleotides need reach only the cytoplasm to have an effect, whereas genetic material that must be transcribed has to enter the nucleus. Genetic vectors have several advantages over oligonucleotides: the level of 'knockdown' of gene expression seems stronger; a treatment may need to be carried out only once, given that the dose is maintained by transcription (in contrast to oligonucleotides, which eventually degrade) and that the vectors are replicated during cell division; and the dose of small RNAs can remain constant even if cells are dividing rapidly.

Most companies, however, have embraced synthetic oligonucleotides, which work more like conventional drugs. "With viral vectors, it's hard to control when they are on and off," Kay says. "With synthetic RNA, you just give the dose, and when it's gone, it's gone." But even getting enough RNA (or, in some cases, DNA or hybrid oligonucleotides) into the cytoplasm is a major challenge. Highly charged and 10 to 30 times heavier than typical small-molecule drugs, oligonucleotides do not readily enter cells. In addition, nucleases chop them into fragments, and they are quickly excreted in the urine.

Some companies are exploring encapsulation technologies to solve these issues. In this approach, oligonucleotides are sequestered in various kinds of nanoparticle to further protect them from degradation and to direct them to the appropriate tissues.

This March, researchers from the California Institute of Technology and Calando Pharmaceuticals (a subsidiary of Arrowhead Research), both in Pasadena, California, announced an important first for encapsulation technologies: proof of principle in a human patient. The small study showed that nanoparticles packed with short interfering RNAs (siRNAs) and injected into a patient's blood delivered their cargo to melanoma cells, silencing the

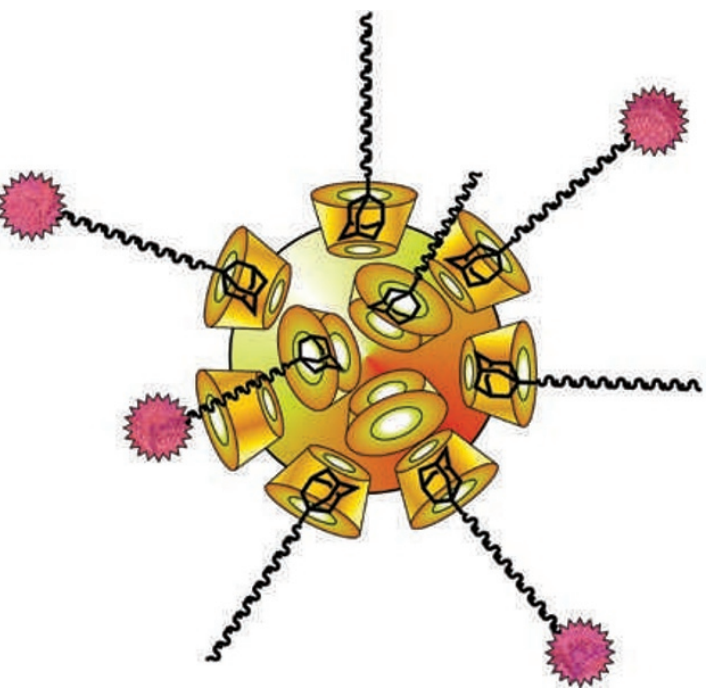

says John Maraganore, chief executive of Alnylam. "We've chosen to develop a range of strategies."

\section{The right molecule}

The downside of delivery vehicles is that they add complexity and manufacturing costs. Their components must be thoroughly vetted in terms of intellectual property and potential toxicity, and figuring out how to mix, measure and deliver both RNA molecules and nanoparticles is far from trivial.

An alternative strategy is to pursue technologies that can bring molecules readily to the therapeutic site for a particular disease without needing such vehicles: injection into the eye or inhalation into the respiratory tract, for example. "Because you can design siRNAs to any sequence, it opens up so many possibilities that choosing a disease indication and even a target becomes very complicated," says Pamela Pavco, vice-president of phar-

targeted gene ${ }^{2}$. These nanoparticles, similar to those of other companies, have separate components for encasing RNA, targeting specific cell types, promoting stability and preventing aggregation, each of which is highly engineered and optimized.

The list of encapsulation strategies being tested is long. RXi Pharmaceuticals in Worcester, Massachusetts, is using components of yeast cell walls. Tekmira is working on stable nucleicacid-lipid particles (SNALPs), which consist of a suite of different lipids encapsulating small RNAs. In March, the company announced a collaboration to evaluate SNALP technology for delivering siRNAs provided by Pfizer, headquartered in New York. The same month, Alnylam announced that siRNAs formulated with a lipid-based coating it had developed in collaboration with researchers at MIT could silence ten targeted genes simultaneously when delivered to rodents. Which approaches will be successful is still very much up in the air. "We believe there's no specific, single magic bullet,"

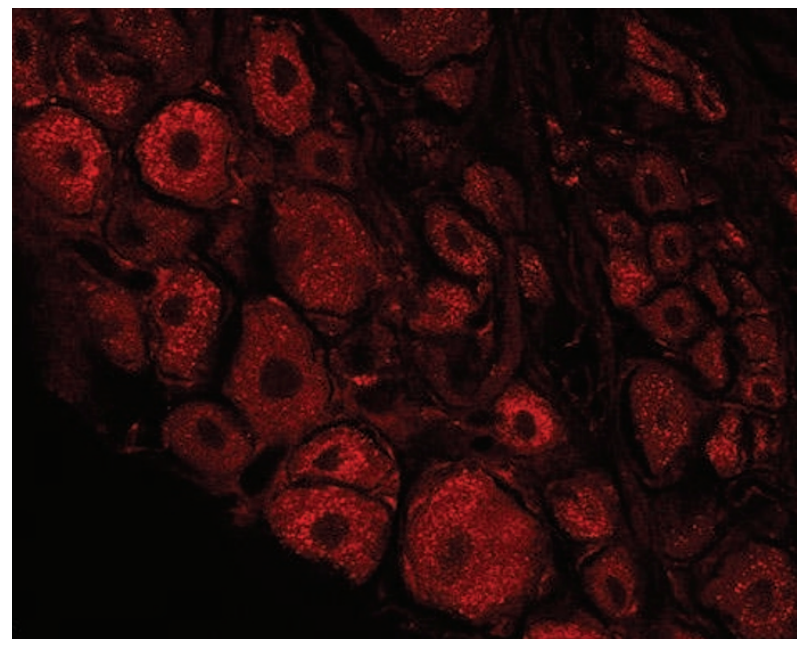

Small RNAs (red) encapsulated in a cationic lipid formulation can enter rat spinal-cord cells and silence their target gene. maceutical development at RXi. "So picking the right path forward becomes very important." Quark Pharmaceuticals in Fremont, California, for example, is the first company to start a clinical trial using siRNAs delivered into the bloodstream; its drug candidate, QPI-1002, is designed to prevent post-surgical acute injury of the kidneys, where RNA molecules are naturally taken up. "We go where the siRNAs go," says James Thompson, vice-president of pharmaceutical development.

Several companies seeking a way to deliver small RNAs are pursuing the strategy of modifying the RNAs themselves so that they will reach their target. "The advantage is that you are working with one compound, so there is no formulation," says Anastasia Khvorova, chief scientific officer at RXi, of her company's most recent development programmes. "We are trying to bring all the components that you need onto the molecule."

Almost all small RNAs that are introduced into cells or animals have been chemically modified. The modifications can protect the molecules against degradation by nucleases, minimize the off-target effects, increase the potency of the knockdown, and avoid the triggering of immune responses that normally protect against viruses. The types and combinations of such chemical modification are endless but often proprietary.

Companies are investigating a variety of oligonucleotides: for example, shorter or longer than their naturally occurring small RNA counterparts; single-stranded, double-stranded or with gaps in the sequence; or alternating between DNA and RNA. Stanley Crooke, chief executive of Isis Pharmaceuticals in Carlsbad, California, thinks that his company might have made as many as 2,000 types of modification, 
studying them in animals in the 20 years since it began studying antisense drugs. Even so, there is a push to identify new and better modifications, and many companies are converging on similar ones. This year, Isis plans to pick clinical candidates with bicyclic additions to the sugars in the nucleotides. With these modifications, nucleotides are positioned such that basepairing becomes more stable, and potency is boosted, Crooke says. MDRNA of Bothell, Washington, acquired the patents for a similar technology, called bridged nucleic acids (BNAs), from Valeant Pharmaceuticals, headquartered in Aliso Viejo, California, this March. Both kinds of modification are similar to Locked Nucleic Acids, produced by Exiqon of Vedbaek, Denmark, and used by Santaris Pharma, which introduce a ring at the 2 ' position of each sugar molecule. Such modifications increase the halflife by an order of magnitude compared with the types of oligonucleotide now in the clinic, says Santaris vice-president and chief scientific officer Henrik Ørum. "We inject these into animals systemically as naked molecules. Once they are in the tissues, they will have a half-life of weeks to many weeks."

Santaris also focuses on making its singlestranded molecules as small as possible, to aid their entry to cells. RXi has also used this strategy, designing 15-nucleotide double-stranded molecules.

Another approach that is in development is to conjugate antibodies or other specific targeting agents directly to the RNA molecules. Biotechnology company Dicerna Pharmaceuticals in Watertown, Massachusetts, is working with 27-nucleotide sequences, based on their proprietary Dicer Substrate Technology, which enter the RNAi-processing pathway earlier than other synthetic counterparts. It has partnered with Kyowa Hakko Kirin, the biopharmaceutical arm of which is based in Princeton, New Jersey, to provide the delivery technology. But like many companies, Dicerna is interested in multiple delivery strategies.

The rise of RNAi therapeutics companies has spurred the development of additional technology to track how oligonucleotides are processed by the body, but high-throughput purification and analysis technologies are not designed for such large molecules with a strong negative charge. Researchers who want to track nucleic-acid metabolites from humans and animals have a "huge problem", says Michael McGinley, bioseparations product manager for Phenomenex in Torrance, California. "You have polar [charged] oligonucleotides floating around in serum that like to bind to things. How are you going to pull them and their metabolites out and separate them from everything else that's there?" In September of last year, Phenomenex began selling kits and other products, such as its Clarity Oligo-MS and Clarity Oligo-RP chromatography columns, to researchers who are running preclinical and clinical studies. These kits provide highthroughput techniques to isolate synthetic oligonucleotides from biological fluids such as serum and urine, as well as from solid tissues, including liver and lung tissue. Although McGinley anticipates tweaking and improving the products over time, he says that they work well with all of the delivery devices, conjugates and modifications tested so far.

\section{Potency potential}

No matter what the approach, the delivery process is easier if researchers can achieve the same therapeutic effect with fewer molecules, so it is crucial to design small RNAs with high potencies. Most therapeutics companies are using a combination of open-access computer programs and their own algorithms to design siRNA sequences that will knock down a target mRNA. Indeed, some computer programs may generate thousands of sequences against a single mRNA. These are then assessed both by scientists and by bioinformatics programs. That's only the first step, says Khvorova of RXi. "All this computational screening is incredibly important, but then you need to do physical screening."

There are significant differences between small-molecule screening and RNA screening, says Caroline Shamu, director of the ICCBLongwood Screening Facility at Harvard

\section{MICRORNAS AS BIOMARKERS}

In the 1990s, Carlo Croce, then director of the Kimmel Cancer Center in Philadelphia, Pennsylvania, was hunting for genes involved in chronic lymphocytic leukaemia. The disease was consistently associated with a lesion in chromosome 13 , and so, back before the human genome was sequenced, his lab determined the identity of the nucleotides in an 800-kilobase stretch from the deleted region and began searching for protein-coding genes. "We failed, for six years," recalls Croce, now director of the Human Cancer Genetics Program at the Ohio State University in Columbus. His lead graduate student left science to go to business school.

After another false start and a lucky conversation, Croce obtained cells from a patient who had leukaemia involving a very small translocation, only about 30 kilobases. Croce examined this region thoroughly enough to convince himself that it contained no genes. Then he read about microRNAs, which had just

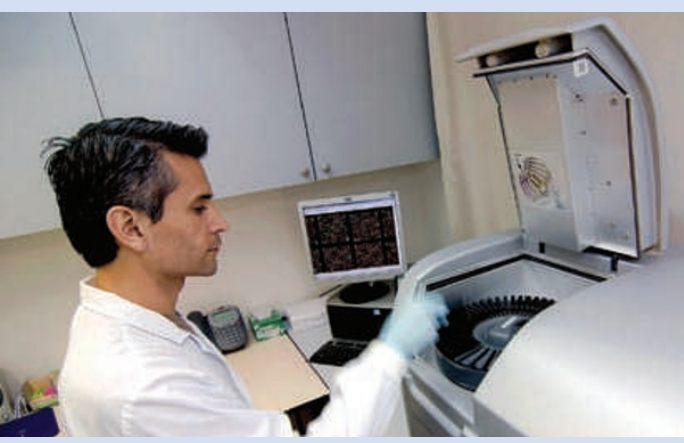

Rosetta Genomics is using microarrays and PCR to identify microRNA biomarkers and develop tests.

been discovered in mice. Further experiments quickly revealed that the region encoded two microRNAs, which, in 2002, were the first to be implicated in disease 4 .

A few years later, researchers led by Todd Golub at the Broad Institute in Cambridge, Massachusetts, examined RNA molecules in tumours and reported that using just a small number of microRNAs, about 200, provided a better classification of tumours by type and source than using 16,000 messenger RNAs ${ }^{5}$. There's great potential, says Croce. "There is no doubt in my mind that microRNA can be used for diagnostic and prognostic purposes."

Indeed, although insurance companies will not yet pay for them, at least two companies offer services that involve testing for microRNAs in biopsies from patients with cancer: Rosetta Genomics in Rehovot, Israel, and Asuragen in Austin, Texas.

Such tests are possible because microRNAs are surprisingly stable both in the body and in paraffin blocks, says Muneesh Tewari, a researcher at the Fred Hutchinson
Cancer Research Center in Seattle, Washington, and lead author on one of the first papers showing that microRNA can be extracted from plasma and serum ${ }^{6}$. "The hard part of this," he says, "is working with small quantities of starting RNAs and applying this to the technologies" that can identify them. Once extracted, known microRNAs can be identified by using microarrays and quantitative PCR; both known and unknown microRNAs can be identified by sequencing.

But finding microRNAs in samples from patients is only the first step to identifying which microRNAs carry information about disease, Tewari says. "Very little is known about the variation of microRNAs." The results of experiments can vary for reasons besides disease, says Jun Lu, a genetics researcher at Yale University in New Haven, Connecticut, and first author on the paper with Golub ${ }^{5}$. But, he says, "if it's a strong discriminator, then you should get the same answer no matter what platform you're using". 
Medical School in Boston, Massachusetts. Small-molecule drugs are often screened against proteins in solution, whereas RNAs are screened in cells. So the results of RNA screening are more variable and take much longer to obtain.

The most important consideration, says Shamu, is the use of controls. Researchers have a plethora of protocols that are appropriate for screening small molecules against proteins, in which most compounds will show no activity, she says, but many of these strategies cannot be relied on for screening siRNAs. Different siRNA sequences targeted to
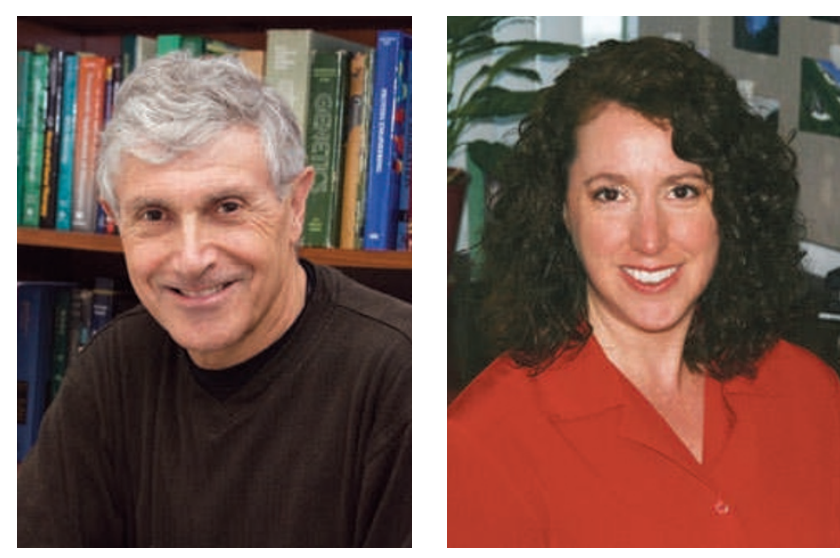

John Rossi and Aimee Jackson are pioneers in the field of RNAi.

has been selected by evolution. It's a gentler way of bringing about a therapeutic benefit."

Although microRNAs have complex biological effects, Jackson says that some aspects of using them are simpler. For siRNA, the targets are transcripts, mRNA molecules that could be several thousand nucleotides in length and part of populations that encompass several splice variants. For microRNA-based therapies, the goal is either to knock down or to replace a particular natural microRNA that is fewer than two dozen nucleotides long.

The surprises have not stopped

of research and development at Thermo Scientific Genomics, part of Thermo Fisher Scientific. Later this year, his company will provide lentiviral vectors for the delivery of microRNA mimics in vivo, and Leake says that researchers are already using microRNAs in vivo. Perhaps as many as one-third of his customers are using or planning to use the products in animals such as mice, rats and guinea pigs. And this March, researchers led by Robert Weinberg at MIT showed therapeutic proof of principle for targeting microRNAs, in a mouse model of metastasis ${ }^{3}$.

Several companies are already developing microRNAs for treating disease, as well as for diagnosing disease (see 'MicroRNAs as biomarkers'). Established companies such as Santaris have brought microRNAs into their discovery and development programmes. In 2007, Alnylam and Isis pooled their expertise and intellectual property to launch Regulus Therapeutics in Carlsbad, California, another company devoted to microRNAs. Since then, Regulus has announced collaborations with drug giant GlaxoSmithKline, headquartered in Brentford, UK, to test microRNAs for treating inflammatory diseases and hepatitis $\mathrm{C}$ virus infection.

The finding that different siRNA constructs diminish the activities of different sets of offtarget genes was made by Aimee Jackson, when working at the now-defunct Rosetta Inpharmatics, a subsidiary of Merck that was based in Seattle, Washington. This effect is now attributed to siRNAs acting as microRNAs and is often controllable through specific chemical modifications. Although researchers are understandably cautious about pursuing constructs that can affect numerous gene products, Jackson is one of many scientists intrigued by the therapeutic potential of microRNAs, an interest that prompted her to move to Regulus as its director of drug discovery. The idea of using microRNAs to fight disease is similar to using combination therapies that block tumour-cell proliferation or HIV replication by targeting several proteins in a pathway, she says. In fact, compared with the sharp, single-target knockdown achieved with siRNA, she thinks that the broader, more moderate effects of microRNAs may be an advantage. "It's a combination that with the discovery of siRNAs and microRNAs. Some microRNAs isolated from cells, for example, display a heterogeneity in sequence length that Jackson thinks might reflect molecules with different functions encoded by the same microRNA transcript, requiring researchers to further investigate which is the best target.

Such discoveries mean that companies must be vigilant in looking for unanticipated routes for toxicity in their preclinical and clinical testing. "My feeling is that you're never going to be able to predict all the off-target effects you might get," says John Rossi, one of the founders of therapeutics companies Dicerna and Calando and chair of molecular and cellular biology at the Beckman Research Institute of the City of Hope.

But the flip side of this uncertainty is excitement: as is the case for siRNA, the discovery of worrisome off-target effects might be a window onto cellular machinery that could be harnessed for therapeutic effect. Rossi says that it's becoming routine to find RNAs doing surprising things in surprising places.

One aspect that's changing fast is the numbers and types of small RNA being uncovered, says Leake, who develops tools for Thermo Fisher Scientific. He notes the emergence of molecular classes such as long non-coding RNAs and transfer-RNA-derived small RNAs. "Non-coding RNA in general is becoming a widely studied area, so we firmly believe these tools will be applicable to other RNAs."

Quark Pharmaceuticals' James Thompson also marvels at the changing directions in the field. A veteran of the RNA field, he is, like many, baffled at how long it took to notice the importance of tiny RNAs. "The RNA machinery is as abundant in cells as are ribosomes, and yet we never saw it. We didn't see this coming," he says. "Who knows what's coming next."

Monya Baker is technology editor of Nature and Nature Methods.

Fire, A. et al. Nature 391, 806-811 (1998).

2. Davis, M. E. et al. Nature doi:10.1038/nature08956 (2010).

3. Ma, L. et al. Nature Biotechnol. 28, 341-347 (2010).

4. Calin, G. A. et al. Proc. Natl Acad. Sci. USA 99, 15524-15529 (2002).

5. Lu, J. et al. Nature 435, 834-838 (2005).

6. Mitchell, P. S. et al. Proc. Natl Acad. Sci. USA 105 10513-10518 (2008)
People are taking microRNAs into living animals, as well as cells, says Devin Leake, director 